\title{
Editorial Signs
}

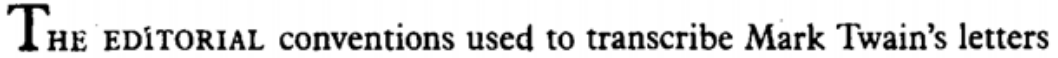
were designed, in part, to enable anyone to read the letters without having to memorize a list. The following is therefore offered less as a necessary preliminary than as a convenient way to look up the meaning of any convention which, in spite of this design, turns out to be less than self-explanatory. Only the editorial conventions used in this volume are given here, since each new volume will require a slightly different list. New or newly modified conventions are identified by an asterisk $\left({ }^{*}\right)$. Not included are the typographical equivalents used to transcribe Mark Twain's own signs and symbols in manuscript. For those equivalents, and for a more discursive explanation of editorial principles, see the Guide to Editorial Practice, pp. 695-722.
\end{abstract}

\section{Editorial HeAding}

From ... Clemens is named in the heading only when he wrote jointly with someone else.

... with a Used when two persons are addressed in the same letter, note to ... but Clemens intended the second to read only the briefer part, or "note."

per... Precedes the name or identity of the amanuensis or agent who inscribed the document sent or received.

2? May On this day-give or take a day.

1-3 May On any day (or days) within this span.

1 and 2 May On both days. 
The source document is the original letter, almost invariably Clemens's holograph manuscript.

(damage emended) The source document has sustained significant damage, and the transcription therefore includes, without brackets, emendation to restore the affected text.

(MS facsimile) The source document is a photographic facsimile of the MS, not the MS itself.

(Paraphrase) The source document preserves some of the words of the original letter, but is manifestly not a deliberate transcription of it.

(Transcript) The source document is a printed, handwritten, or typed (TS) transcription of the letter, not necessarily made at first hand.

\section{LetTer Text}

NEW-YORK Extra-small small capitals with no initial capitals signify printed text not originated by Clemens, such as letterhead or the postmark.

SLC) Italicized extra-small small capitals within an oval border transcribe monograms or initials printed or embossed on personal stationery.

Feb. 13, Text above a dotted underscore was inscribed in a printed blank in the original document.

. . . Editorial ellipsis points (always centered in an otherwise blank line) signify that an unknown amount of the original letter is judged to be missing.

Ruled borders are an editorial device to represent the edge of a document, usually printed or partly printed, such as a telegram blank or newspaper clipping.

two cance- Cancellation is signified by slashes for single characters deletions, (and underscores), rules for two or more characters. 
Well, I pass. A hairline rule signifies a mock, or pretended, cancellation: words lightly and distinctively crossed out, easily read, and often still necessary to the sense.

marking it up . Insertion is signified by a single caret for single characters, two carets for two or more characters.

shaded words Gray background identifies text originated and inscribed by someone other than Clemens-or by Clemens himself when he altered or signed what his amanuensis wrote. ${ }^{*}$

[ ] Author's square brackets are transcribed this way to avoid any confusion with editorial brackets.

Editorial square brackets enclose [editorial description]; words or characters omitted by the writer an[d] now interpolated by [the] editors; and text modified by description, such as [in margin: All well].

siamond The diamond stands for a character, numeral, or punctuation mark the editors cannot read because it is physically obscured or obliterated. It never stands for the space between words.

double $=\quad$ The hyphen is to be retained. Single hyphens at the ends hyphen of lines therefore signify division only.

$\mathrm{Sam}^{t}$

Superscript ell is always italicized to prevent confusion between one $\left(^{1}\right)$ and ell $\left({ }^{1}\right)$. The sign $\ldots m$. transcribes a paraph or flourish.*

The envelope and full-measure rule signal that everything transcribed below them was written, stamped, or printed on the envelope or on the letter itself at the time of transmission or receipt.

Signifies the end of a line in the source document. 
